\title{
Journal of Stem Cell and Regenerative Biology
}

\section{Double Edge Effect of DPP4 Inhibitor Sitagliptin, A Type-2 Anti-Diabetic Drug, on Inflammation, Injury and Cancer}

\author{
Mrinmoy Sarkar, Sananda Dey, Biplab Giri*
}

Experimental Medicine and Stem Cell Research Laboratory, Department of Physiology, West Bengal State University, Kolkata, India

*Corresponding author: Biplab Giri. Experimental Medicine and Stem Cell Research Laboratory, Department of Physiology, West Bengal State University, Kolkata-700 126, India; E-mail: bgiri.emscrl@gmail.com

\begin{abstract}
Maintaining a healthy glycaemic condition is associated with so many more other protective/precautionary attributes. Recent advancement in the field of drug discovery have led us find more and more options to choose from. DPP-4 inhibitor sitagliptin makes sustained expression of incretins GLP-1 and GIP that in turn establishes glycaemic control. But recent reports claimed pro-inflammatory as well as pro-carcinogenic action of the drug after prolonged use in T2DM patients. Interestingly, in those cases also, the effector molecule responsible is said to be the incretin GLP-1. The same molecule has been hypothesized for being responsible to cause thyroid C-cell carcinomas. In vitro investigations using human cell lines did not show any clues in support of carcinoma formation. Instead, the DPP-4 inhibitory action of sitagliptin serves in other complications associated with T2DM. In any tissue injury due to hypoxic stress in T2DM, sitagliptin serves in the tissue repair mechanisms via its DPP-4 inhibiting property. Consequently, DPP-4, for not being able to be active in presence of sitagliptin, fails to truncate the CXCL-12/SDF-1 and therefore helps in the homing of HPCs/EPCs to repair the injured tissue. In conclusion, it can be said that more research is definitely required to be confirmed about the pre-cancerous activity of the drug, and to make the drug more usable in favour of the other beneficial effects of it.
\end{abstract}

Keywords: DPP-4; Sitagliptin; Diabetes; Cancer; Inflammation; Stem Cell
Received Date: July 12, 2016

Accepted Date: July 15, 2016

Published Date: July 20, 2016

Citation: Sarkar, M., et al. Double Edge Effect of DPP4 Inhibitor Sitagliptin, A Type-2 Anti-Diabetic Drug, on Inflammation, Injury and Cancer. (2016) J Stem Cell Regen Biol 2(2): 102- 108.

DOI: $10.15436 / 2471-0598.16 .017$

\section{Introduction}

Today's biggest concern in human lifestyle is maintaining a decent health that has been turning into a very challenging job due to the increase in the global diabetic population. In spite of the fact that globally under-nutrition is widespread, especially in the $3^{\text {rd }}$ world countries, contrasting views can be captured in more developed countries where there is habitually disproportionate food consumption leading to obesity and diabetes, cardiovascular disease and often some or the other forms of cancer. Among the mentioned diet-associated complications, obesity and diabetes and the cancer are well documented to be linked with each other ${ }^{[1,2,3]}$. Cancer has been linked with diabetes only recently though. Inflammation has been a major problem in the patients with diabetes. Apart from that, the major linked pathological conditions include cardiovascular pathology, diabetic nephropathy, retinopathy, neuropathy and others. The best pre-emption to resist the advancement to the diseased condition is a proper glycaemic control ${ }^{[4,5]}$. The effective treatment of diabetes merely came to be plausible in the 1920s with the discovery of insulin. Insulin came handy in combating type-1 diabetes mellitus (T1DM) while it is also recommended for prevention of type-2 diabetes mellitus (T2DM) in cases where oral drugs stand insufficient or during particular conditions like pregnancy, perisurgical time or in acute states (myocardial infarction $)^{[4,5]}$.

But there are conditions when use of insulin cannot be prescribed to control hyperglycaemia. Since insulin is a potential 
growth factor for mammalian cells, it would have a boomerang action leading towards the initiation of tumorigenesis or carcinogenesis. There are dozens of published data and analysis that emphasize the association of cancer with diabetes, the disease itself, as well as the treatment of $\mathrm{it}^{[2,6,7]}$. This, in fact, was a unanimously accepted consensus by the experts of American Diabetes Association and the American Cancer Society ${ }^{[1,2]}$. Epidemiological data supports the view that diabetics remain in a significantly higher risk of cancer $^{[2]}$.

Contemporary oral treatment modalities are targeted to reduce the hepatic glucose yield, easing glucose absorption, increasing peripheral glucose utilization, and stimulating the release of insulin. Altogether, the treatment regime is involved in the management of hyperglycaemia associated with T2DM but the pathophysiological decline in the beta-cell function and mass largely remain unaffected and the gap in knowledge remained over the long term. These agents- including biguanides, sulfonylureas, meglitinides, alpha-glucosidase inhibitors and thiazolidinediones -are also often concomitant by substantially inappropriate side-effects ${ }^{[8]}$.

Sitagliptin, introduced in 2006, was the first FDA approved drug in the Dipeptidyl peptidase-4 (DPP-4) inhibitor class. This group of therapeutic agents is considered unique in its target mechanistic approach in the treatment of diabetes and thus to improve glycaemic control.

\section{Classification of the drug}

Sitagliptin (Januvia, Merck) belong to the DPP-4/CD26 inhibitor class of insulin sensitizers which are anti-hyperglycaemic agents directed towards improvement of glycaemic control in patients with T2DM. The drug (sitagliptin) is available as tablets of25-, 50-, and $100 \mathrm{mg}$. The drug has been used as monotherapy as well as combination therapy (along with other class of anti-diabetic drugs) intending to achieve synergistic efficacy. For example, a sitagliptin and metformin combination (Janumet, Merck) was made available and approved in $2009^{[9]}$.

\section{Pharmacology and mode of action}

Modern day research in regard to the management of T2DM and hyperglycaemia has led to new targets in connection with the role of gut hormones in the regulation of pancreatic beta cells. After the ingestion of meal, a club of hormones are secreted from the intestinal mucosa, i.e. incretins [glucagon-like peptide (GLP-1), and glucose-dependent insulinotropic peptide (GIP) etc.], causing stimulation to the beta cells resulting in release of insulin ${ }^{[10,11]}$. Reasonably, this insulin release is glucose dependent. GLP-1 is secreted from the L-cells of distal small intestine after food intake and GIP is mainly secreted by K-cells of proximal small intestine ${ }^{[11]}$. After the action, GLP-1 gets degraded by DPP-4, a cell surface serine protease which cleaves dipeptides, containing proline or alanine in $\mathrm{N}$ terminal position ${ }^{[12]}$. Taking under consideration the anti-diabetic properties of GLP-1 receptor agonists (GLP-1 RA), couple of drugs, namely exenatide and liraglutide, was developed to exploit the advantageous incretin pathway through subcutaneous administration for prolific secretion of insulin. Another way to utilize the incretin system is by inhibiting the DPP-4 enzyme by oral administration of sitagliptin, vildagliptin, saxagliptin, linagliptin or alogliptin. Incretin therapy has certain associated health benefits like bodyweight reduction and dodging from hypoglycaemic attack ${ }^{[13]}$.

GLP-1 acts through its G-protein coupled receptor pathway. The incretin stimulates the propagation and differentiation of pancreatic alpha and beta cells by increasing the synthesis of insulin. This is achieved via giving stimulus to the insulin promoter factor 1 (Pdx-1, pancreatic and duodenal homeobox 1) ${ }^{[14]}$. Apart from increased insulin secretion, GLP-1 can act to achieve glycaemic control via several routes, viz a decline in the glucagon concentrations, a delay in the gastrointestinal clearing, and potential induction of satiety. GLP-1 plays a role in the decreased rate of apoptosis of pancreatic beta cells other than assisting proliferation of the same population ${ }^{[15]}$. Circulating levels of GLP-1 gets elevated immediately after a meal only to decrease in a couple of minutes (half-life $<2$ minutes) when it is rapidly inactivated by DPP-4, a proteolytic enzyme. DPP-4 inhibitors like sitagliptin have sealed that gap to increase the activity by the incretins like GLP-1.

\section{Sitagliptin and inflammation}

Sitagliptin heals: Inflammation (originally Latin word, meaning setting on fire) is generally defined as a confined tissue response towards infection or wound which is categorized by the four basic signs- heat (calor), redness (rubor), swelling (tumor) and pain (dolor ${ }^{[16]}$. Apart from these signs, there are certain molecular markers by which the inflammation can be analysed in living systems.

As part of our concern, diabetes and inflammation are linked together hugely. The foremost feature of T2DM is the presence of higher concentration of glucose in the peripheral blood. Prolonged exposure of beta cells to the elevated concentration of this glucose and fatty acids results in upsurge in the metabolic activity of the islet cells, thus in turn giving rise to increased production of reactive oxygen species (ROS) ${ }^{[17]}$. ROS is vastly responsible for inflammation. Other than this, there are certain pro-inflammatory molecules that mediate inflammation. DPP-4 (CD-26) is present in the cell surface which is a molecule of this kind. Therefore, inhibitor of DPP-4 is a potent anti-inflammatory molecule. Reports have been there regarding the use of sitagliptin to combat the inflammatory activity of DPP-4 by inhibiting its synthesis and the action there of. The use of sitagliptin has been reported to be very advantageous in the treatment of atherosclerosis, a chronic inflammatory disease, because of the drug's potent and rapid anti-inflammatory effect ${ }^{[18,19,20]}$. Exenatide, a potent GLP-1 agonist had also been observed to show a similar act and thus protecting against inflammation ${ }^{[21]}$.

Therefore, by inhibiting its synthesis or inactivating the DPP-4 the bioavailability of GLP-1 can be extended to exert its beneficial effect against inflammation ${ }^{[21]}$. Apart from this finding, sitagliptin usage interestingly reduced the free fatty acids (FFA) concentrations in the plasma and thereby improving the insulin sensitivity. The fall in the concentration of the FFA resulted in a 
decrease in inflammation, which is in line with the finding that FFA induces inflammation ${ }^{[2]}$. Using mononuclear cells (MNC), sitagliptin had been shown to reduce the intranuclear binding of NF- $\mathrm{kB}$ and decrease the expression of certain other pro-inflammatory molecules like TNF-alpha, TLR-4, CD-26 ${ }^{[18]}$. TLR-4 is a receptor for the endotoxin lipopolysaccharide (LPS). It becomes evident from the above information that LPS induced inflammation can be checked by sitagliptin ${ }^{[18,23]}$. Human MNC data was potentially supported by rat ventricular myoblast cell line experiments data only to show that like in mononuclear cells of humans, in the cardiomyocytes of rats the NF- $\mathrm{kB}$ is downregulated and inflammatory response is reversed as a consequence ${ }^{[18]}$. Apart from playing roles in reduction of inflammatory responses in blood cells and cardiomyocytes, the anti-diabetic drug raised strong to be effective enough in the treatment of diabetic nephropathy (DN), an associated complication in diabetics. A diabetic rat model demonstrated the drug's efficacy to inhibit the inflammation and associated pro-apoptotic mechanism of the kidney cells in vivo ${ }^{[24]}$ (Figure 1).

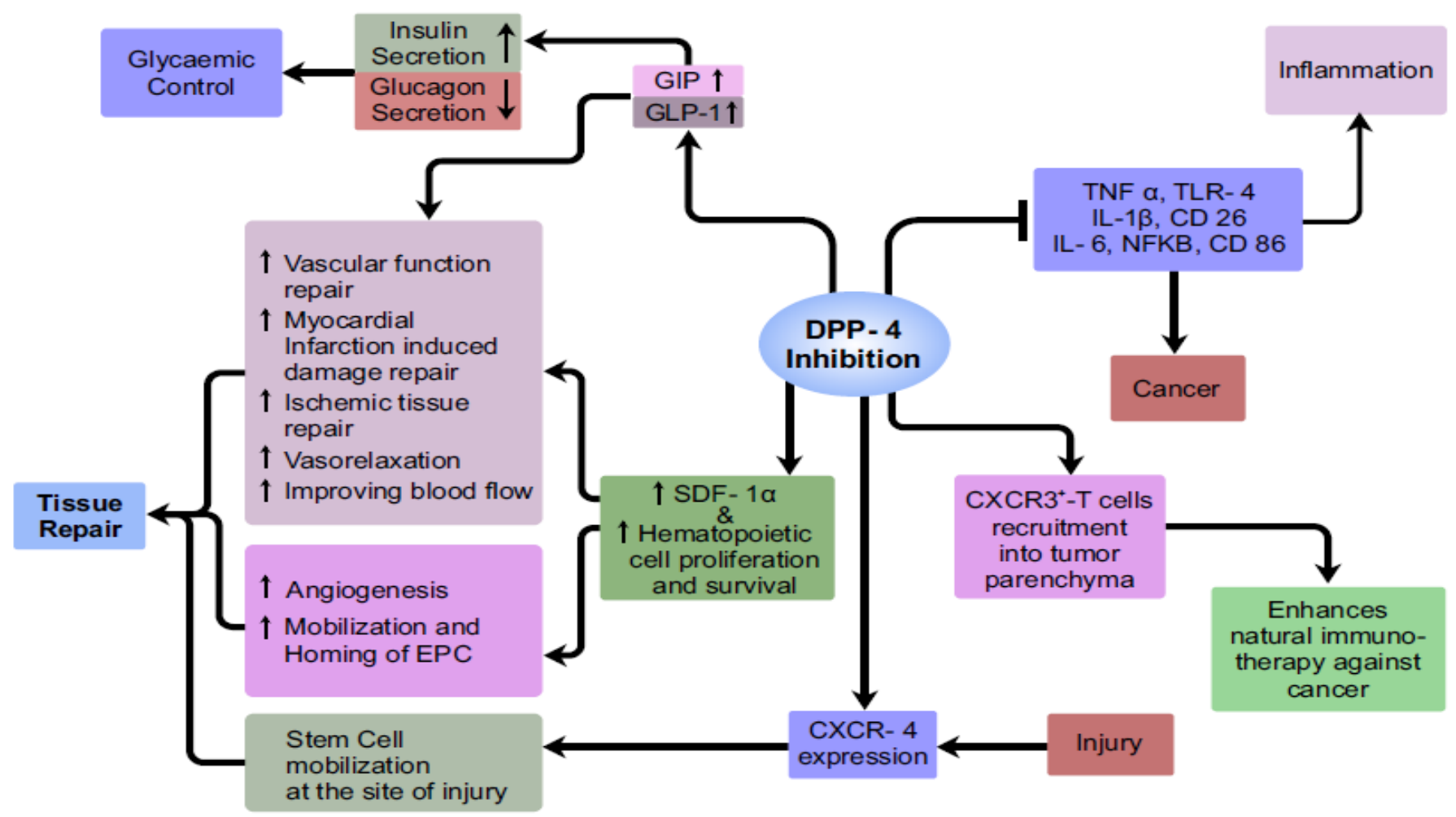

Figure 1: Depicting various roles played by a DPP-4 inhibitor sitagliptin in sustaining glycaemic control, tissue repair, enhancement of immunotherapy against cancer and anti-inflammatory mechanisms.

Some contradictions: Nearly half a dozen scientific reports have stated about the adverse effects of using agonists of GLP-1 pathway. Sitagliptin is one of them. It has been reported recently that using sitagliptin for long duration resulted in increased rate of acute inflammation of the pancreas resulting in pancreatitis ${ }^{[25,26,27]}$. Again, a study by Suissa et al, reported no significant association between pancreatitis and sitagliptin usage as compared to other medications ${ }^{[28]}$.

Other adverse effects, reported from post-marketing analysis of sitagliptin, include- serious allergic reactions, including anaphylaxis, angioedema, and Stevens-Johnson syndrome. It has been reported by the Food and Drug Administration (FDA), USA, that sitagliptin use may induce fatal/non-fatal necrotic pancreatitis in addition to acute pancreatitis ${ }^{[2,29]}$. Apart from these, recent studies on case reports have raised some contradictions that DPP-4 inhibitor sitagliptin may be responsible for acute and chronic inflammatory diseases like rheumatoid arthritis/ arthralgia, an inflammatory pain in the joint and psoriasiform, one type of autoimmune skin inflammation ${ }^{[30,31]}$.

In spite of all these studies based on population data and case reports, the adequacy in this regard has not been achieved till date. Since the population based studies have certain limitations and the reasons behind the initiation of inflammatory response has not been experimentally assessed, the thrust for knowledge remained on the issue.

\section{Sitagliptin and cancer}

Resulting from the upsurge in the scientific investigations regarding the association of diabetes and cancer, several anti-diabetic drugs have been put under trial to evaluate their anti-cancer therapeutic efficacy. Sitagliptin, besides being recognized to be a potent anti-inflammatory agent in different studies, mentioned in this manuscript, has been observed to cure cancer also. In this section, we would scrutinize the risk factors, if any, of using sitagliptin in cancer-diabetic patients and of course the benefits of inhibiting DPP-4 enzyme in the anti-cancer mechanisms.

Anti-cancer activity: Data are scarce in regard to studies evaluating the role of sitagliptin or other DPP-4 inhibitors in carcino- 
genesis or tumorigenesis. There are very few evidences suggesting anti-cancer role of the drug. A similar in vitro study with colon cancer cells (HT-29) reports the drug's anti-cancer property with a very low IC-50 value. A higher IC-50 was observed in case of another DPP-4 inhibitor, vildagliptin ${ }^{[32]}$. An earlier study had shown that the inhibition of growth of the colon cancer cells as well as induction of apoptotic cell death (ACD) mechanism is carried out by the incretin GLP-1 after the activation of its receptor ${ }^{[33]}$. A very recent study from our laboratory also demonstrates a similar result in case of HCT-116 colon cancer cells and HepG2 hepatocarcinoma cell line (data not shown; unpublished). But, in their article by Amritha et al (2015), the anti-pancreatic cancer activity of the DPP-4 inhibitor was mentioned, though this is certainly arguable keeping in mind the recent scientific reports. Keeping aside the GLP-1 mediated action of the medicinal agent, investigators found some evidence that supports the drug's action causing the body's own defence system to get activated and killer /effector lymphocytes can invade the tumor and inflammation territory to kill the cancerous cells. The anti-cancer activity is said to be carried out depending upon the presence or absence of DPP-4 or CD-26 on the cell surface. Sitagliptin inhibits the DPP-4 and thus biologically active chemokine CXCL10 is stabilized and CXCL10 mediated CXCR3 + T cells recruitment in to the tumor parenchyma enhances $\mathrm{T}$ cell dependent antitumor effect. Therefore, the study validates that the use of sitagliptin may augment the general and adjuvant based immuno therapy of cancer ${ }^{[34,35]}$. (Figure 1)

Pro-cancerous motion: The drug, in several population based studies, was found to be responsible for development of cancer. As stated earlier in the text, sitagliptin has been found to trigger pancreatitis and pancreatic cancer after prolonged treatment ${ }^{[25,26,27]}$. A study involving Taiwanese T2DM patients, suggested a possible link between sitagliptin use and thyroid cancer ${ }^{[25,36,37]}$. Generally speaking, thyroid cancer is a rare disease and it requires prolonged exposure to the drugs with higher bioavailability and longer half-life to promote the proliferating pathway ${ }^{[38]}$. In rodents, GLP-1 agonists have been found to elevate the risk of C-cell adenoma and medullary carcinoma spontaneously while in the humans it is thought to be rare. There have been reports that cell lines from the C-cell origin of rodents, showed elevated production of cAMP second messenger and instant increase in secretion of calcitonin ${ }^{[39]}$. The similar cells from the human origin had also been tested to find if any proliferative mechanism does act in the same line. But human origin cells, though exposed to a far higher concentration of the GLP-1 agonists, did not give any indication of becoming cancerous $^{[40]}$. Various similar studies were carried out in the run and they all demonstrate the effect of GLP-1 protein itself or, GLP-1 agonists upon GLP-1 receptors or effects of GLP-1 receptor agonists. Our drug of concern, sitagliptin is a DPP-4 inhibitor and is very faintly and not directly related to the mode of action of aforesaid effector molecules.

\section{Sitagliptin in injury and tissue repair}

Apart from the drug's debated influence in the anti-inflammatory mechanisms and several other cases involving anti-cancer instruments, this inhibitor of DPP-4 enzyme extends its activity to enhance the homing and engraftment of the hematopoietic stem cells (HSCs). Kim et al (2014) demonstrated in one of their contemporary investigation that the DPP-4 inhibitors have pivotal effects on the hematopoietic system ${ }^{[12]}$. This study supported a previous investigation by Gupta et al (2012), suggesting anti-platelet action of sitagliptin in both diabetic and healthy persons ${ }^{[41]}$. Kim et al (2014) suggested with evidence that the recovery from bone-marrow suppression was enhanced with the use of DPP-4 inhibitor ${ }^{[12]}$. It was explained in a recent report that DPP-4/CD26 has potential influence on the modulation of hematopoietic chemokine effects. The study illustrates the effects of the proteolytic enzyme DPP-4/ CD26 that can influence the activity of certain proteins by forcing their post-translational modification. A truncated CSF (T-CSF) fails to stimulate the proliferation of hematopoietic progenitor cells (HPCs). Truncated CXCL-12 (T-CXCL-12) is another such protein which is inactivated by the DPP-4 enzyme. The most harmful result, among what they deliver is the inhibitory action of the truncated versions over the expression of their full length protein variants ${ }^{[42]}$.(Figure 1)

The CXCL-12 is one of the substrates of DPP-4 as we know. The other terminology used to indicate CXCL-12 is SDF-1 (Stromal-cell Derived Factor-1). Among all other substrates of DPP-4, SDF-1 has been studied most intensely. SDF-1 has 2 variants - alpha and beta, and works as a homing molecule for hematopoietic stem cells (HSCs), hematopoietic progenitor cells (HPCs) and endothelial progenitor cells (EPCs). The upregulation of expression of SDF-1 takes place in case of injury. Other than this the homing molecule is expressed constitutively by the bone marrow cells ${ }^{[12,43]}$. Whenever any vascular injury is occurred, in response to the SDF-1 generated from the injury sites, EPCs get bound to mobilize to that sites through blood stream and put back together the anatomical integrity of the injured tissue ${ }^{[44]}$. In presence of DPP4 inhibitor, concentration of SDF1 rise thereby increasing peripheral blood flow, angiogenesis, and endothelial progenitor cell homing and wound healing ${ }^{[45]}$. It has been reported that interaction between SDF-1 from the hypoxic sites and the receptor of SDF-1/CXCL-12, CXCR-4, on EPCs seems significant in vascular repair and neo-angiogenesis. That an anti-DPP-4 treatment can improve cardiac homing of stem cells and improve heart function after a myocardial infarction (MI) has already been shown in some preclinical studies ${ }^{[46]}$. Therefore it can be clearly understood that treatment of T2DM patients with sitagliptin may enhance amelioration from any tissue injury (including the most common in such patients - cardiovascular injury) along with sustained glycaemic control. (Figure 1)

The DPP-4 does have even more to offer for the scientific society. A new field of interest developed for the investigators after the report of DPP-4 being involved in the list of cancer stem cell markers for colon cancer cells due to having germ-line polymorphisms ${ }^{[47]}$. Forthwith a hypothesis can be drawn that inhibition of DPP-4 by sitagliptin could hypothetically lead to a decreased number of cancer stem cells and the chance of recurrence can be reduced henceforth.

\section{Discussion and Conclusion}

Present studies are targeted towards finding out important and truly efficient drugs in the treatment of diabetics suffering from cancer. Since, different drug action requires different effector molecules to exert the beneficial outputs, it is necessary to have a 
sound knowledge regarding the pharmacology and associated side effects of the drug. The anti-hyperglycaemic action of sitagliptin is beyond doubt. The anti-inflammatory action claims larger use of the drug. The anti-cancerous physiognomies claim the drug's usage irrespective of patients' cancer history. In principle, there could be weak or strong evidence, either in favour of or against the claims that sitagliptin can someway cause pancreatitis, pancreatic cancer, thyroid cancer, or other cancers or work absolutely to oppose all these circumstances. Our present review is concerned about its claimed hostile actions as depicted by several demographic data representations, and whether it is good or bad to use sitagliptin in diabetes patients with or without a history of any type of cancer and inflammatory diseases.

Whether a drug can cause pancreatitis or any drug can cause pancreatic cancer is interrelated. Lowenfels et al. (1993) in their report stated that chronic pancreatitis enhances the risk factor to result in pancreatic cancer by 26-fold as compared with cases not associated with chronic pancreatitis ${ }^{[48]}$. The underlying roots include genetic susceptibility, alcohol abuse and some drugs ${ }^{[49-51]}$. Once chronic pancreatitis has been recognized, the chronic inflammation and bigger intraductal pressure due to stenosis in the pancreatic duct(s) often may show progression towards pancreatic carcinoma ${ }^{[49-51]}$. But, interestingly there are very few or absolutely no data supporting the establishment of chronic pancreatitis associated with treatment of sitagliptin. This is of importance that substantial evidences prove the episodes of acute pancreatitis instead of the chronic one.

Instead, sitagliptin has several times been reported to act against inflammatory mechanisms in past as mentioned in the manuscript. It decreases the risk of LPS induced inflammatory markers like TNF- $\alpha$, ROS, IL- $1 \beta$, IL-6. Apart from this, a major anti-inflammatory action is completed by reducing the activation of NFKB, whereas, it's DPP-4 inhibitory function itself defines its anti-inflammatory action. The inhibitory effect of DPP-4 inhibitor on NFKB also important for its antiproliferative activity against cancer. (Figure 1)

In experiments using transgenic rats who over expressed human islet amyloid polypeptide in the endocrine pancreas (HIP rat), it was demonstrated that except in a very few cases (statistically insignificant) where only sitagliptin was administered no signs of pancreatitis could be traced in the models involving combination therapy of sitagliptin with metformin or metformin alone ${ }^{[24]}$.

Among other aspects of the study, an enhanced rate of ductal cell proliferation was observed in sitagliptin treated group and the condition was ameliorated with the combination therapy with sitagliptin and metformin ${ }^{[24]}$. What we understand from the reports is that sitagliptin usage may bring about ductal cell carcinoma and thereby cause pancreatitis and perhaps in the long run pancreatic carcinoma in rat $^{[24]}$. But in case of humans, it should be the rarest of the cases since the pancreatic cancer is possible only after a long standing insult by chronic pancreatic inflammation.

Present knowledge regarding the known mutation rates and rate of augmentation of cancer causing somatic mutations at respective stages of development of pancreatic cancer suggest that the incidence must be a chronic process, because from a stage of normalcy a duct cell takes about an era (approx. 12 years) to get initiated to be a tumor cell and to give rise to a parental clone from which a pancreatic carcinoma can grow. And these cells then get converted into sub-clones with metastatic capacity in another 7 years and further 3 years from then the disease gets diagnosed with evident clinical symptoms of primary tumor accompanied by metastasis $^{[52]}$. Therefore, the possibility of developing pancreatic cancer via such a process seems a bit difficult from this point of view.

The similar observation was reported in case of development of a thyroid C-cell carcinoma. In experiments, involving rodent animal models, the generation of second messenger cAMP and release of calcitonin were demonstrated to be there behind the upsurge of the C-cell proliferation. In contrary with this, the similar cell lines from human origin did not show similar cyclic AMP (cAMP) generation and cellular secretion thereby denying favouring the same pathway as in case of the rodent model ${ }^{[39,40]}$. Therefore, the reported case of Thyroid C-cell carcinoma needs serious concern to find out the reason behind its development. It cannot be challenged straightaway because a recent investigation with immunohistochemistry suggested that GLP-1 receptors were examined and detected in some, but not all, follicular cells and in some papillary thyroid carcinomas. This indicated the influence of such receptors on the rate of cell proliferation ${ }^{[53]}$. However, contradictory reports do exist which state that GLP-1 receptors are not present outside the C-cells in the thyroid tissue ${ }^{[54]}$.

Other than the above mentioned mechanisms, GLP-1 and its receptor has been reported to enhance the Wnt signalling pathway towards generation of cancer stem cells and high proliferation rates. Although the studies have marked the GLP-1 and its receptor agonists to be responsible for causing cancerous symptoms, but not the DPP-4 inhibitors like sitagliptin ${ }^{[55]}$. Therefore, the present topic demands more discussions to confirm the usability of sitagliptin at the same time, invading the risk of development of cancer.

In agreement with the GLP-1 related studies, reports have been there indicating that a reduced expression of the DPP-4 is associated to malignancy in a few cases like ovarian, colon, and melanoma cancers ${ }^{[56]}$. The mechanism behind the scene might be the viable concentration of GLP-1 due to unavailability of adequate concentration of DPP-4 to degrade GLP-1. So it can be said hypothetically that in these cases also the GLP-1 remained the main molecule to result in progression towards cancer. Hence, the mechanisms related to DPP-4 remained unclear till now.

But research regarding the DPP-4 enzyme and functions related to it, has placed the DPP-4 gene among the markers associated with colon CSC (cancer stem cell) as we have discussed earlier ${ }^{[4]}$. However, DPP-4 activity might also increase in some cancers like hepatocellular carcinoma or skin basal cell cancer and the enzyme's immunosuppressive potency has also been suggested ${ }^{[56]}$. In support of this, Silva et al (2015) have very recently suggested the role of DPP-4 inhibitor in raising a natural immunotherapy against cancer ${ }^{[34]}$. In consistency with this we have previously talked about cases where sitagliptin has been observed to cure colon cancer in vitro ${ }^{[32,33]}$. This in turn justifies the use of sitagliptin to potentially reduce the risk of colon cancer and raise the natural immunotherapy against carcinogenic insult. 
Before declaring the overall conclusion regarding the use of sitagliptin in diabetic-cancer patients, we must not forget about its effects on the hematopoietic cells ${ }^{[12,41,42]}$. In diabetic patients, certain pathophysiological conditions like nephropathy, retinopathy, and cardiomyopathy are seen frequently. In these and other tissue injury cases CXCR $-4^{+}$cells are increased thus activating tissue repair mechanisms. DPP-4 inhibitor, like sitagliptin, enhances the CXCR-4 expression and activity of it by increasing the expression of the ligand of it, SDF-1. This, in turn, is assisting the tissue repair mechanisms in cardiovascular and other tissue injuries by mobilizing and homing of EPC and stem cells. Other than these, the DPP-4 inhibitor enhances vasorelaxation, improves blood flow in organs, and combats hypoxic stresses in tissues by inducing angiogenesis ${ }^{[42,45,46,57]}$. (Figure 1)

After all the discussion, we may conclude that DPP-4 inhibitor (sitagliptin) ensures double edge advantages including anti-inflammatory, anti-cancer and tissue repairing functions, while used by the diabetic populations. Future studies at molecular level are indispensable to reveal more pros and cons of its use.

Acknowledgement: The work is partially supported by the grant of Dr. B. Giri (Grant No. SR/FT/LS-132/2010, SERB, Department of Science \& Technology, Govt. of India). Authours acknowledge Dr. Tuhin Subhra Sarkar for artwork.

\section{References}

1. Dalamaga, M., Diakopoulos, K.N., Mantzoros, C.S. The role of adiponectin in cancer: A review of current evidence. (2012) Endocrine Reviews 33(4): 547-594.

2. Giovannucci, E., Harlan, D.M., Archer, M.C., et al, Diabetes and Cancer: A consensus report. (2010) Diabetes Care 33(7): $1674-1685$.

3. Lewis, J.D., Ferrara, A., Peng, T., et al. Risk of Bladder Cancer Among Diabetic Patients Treated With Pioglitazone: Interim report of a longitudinal cohort study. (2011) Diabetes Care 34(4): 916-922.

4. Aguilar, R.B. Evaluating treatment algorithms for the management of patients with type 2 diabetes mellitus: a perspective on the definition of treatment success. (2011) Clin Ther 33(4): 408-424.

5. N Engl, J. Med. The effect of intensive treatment of diabetes on the development and progression of long-term complications in insulin-dependent diabetes mellitus. The Diabetes Control and Complications Trial Research Group. (1993) 329(14): 977-986.

6. Satija, A., Spiegelman, D., Giovannucci, E., et al. Type 2 diabetes and risk of cancer. (2015) BMJ 350: g7707.

7. Kole, L., Sarkar, M., Deb, A., et al. Pioglitazone, an antidiabetic drug requires sustained MAPK activation for its antitumor activity in MCF7 breast cancer cells, independent of PPARgamma pathway. (2016) Pharmacol Rep 68(1): 144-154.

8. Neumiller, J.J. Differential chemistry (structure), mechanism of action, and pharmacology of GLP-1 receptor agonists and DPP-4 inhibitors. (2009) J Am Pharm Assoc 49(1): S16-S29.

9. Reynolds, J.K. Fixed-dose combination of sitagliptin and metformin for the treatment of type 2 diabetes. (2009) Diabetes Metab Syndr Obes 2: $127-134$.

10. Verspohl, E.J. Novel therapeutics for type 2 diabetes: Incretin hormone mimetics (glucagon-like-peptide-1 receptor agonists) and Dipeptidyl peptidase-4 inhibitors. (2009) Pharmacol Ther 124(1): 113-138.

11. Drucker, D.J., Nauck, M.A. The incretin system: Glucagon-like peptide-1 receptor agonists and dipeptidyl peptidase-4 inhibitors in type 2 diabetes. (2006) Lancet 368(9548): 1696-1705.

12. Kim, N-H., Yu, T., Lee, D.H. The Nonglycemic Actions of Dipeptidyl Peptidase-4 Inhibitors. (2014) BioMed Res Int 2014:368703.

13. Tokajuk, A., Krzyżanowska-Grycel, E., Tokajuk, A., et al. Antidiabetic drugs and risk of cancer. (2015) Pharmacol Rep 67(6): 1240-1250.

14. Drucker, D.J. The biology of incretin hormones. (2006) Cell Metab 3(3): 153-165.

15. Arora, S., Mehrotra, A., Gulati, S.C. Incretins and thiazolidinediones in glucose homeostasis and cancer: role of common polymorphisms. (2012) Cancer Lett 323(2): 128-134.

16. Medzhitov, R. Inflammation 2010: new adventures of an old flame. (2010) Cell 140(6): 771-776.

17. Donath, M.Y. Targeting inflammation in the treatment of type 2 diabetes: time to start. (2014) Nat Rev Drug Discov 13(6): 465-476.

18. Lin, C.H, Lin, C.C. Sitagliptin attenuates inflammatory responses in lipopolysaccharide-stimulated cardiomyocytes via nuclear factor- $\kappa B$ pathway inhibition. (2016) Exp Ther Med 11(6): 2609-2615.

19. Best, C., Struthers, H., Laciny, E., et al. Sitagliptin Reduces Inflammation and Chronic Immune Cell Activation in HIV+ Adults With Impaired Glucose Tolerance. (2015) J Clin Endocrinol Metab 100(7): 2621269.

20. SatohAsahara, N., Sasaki, Y., Wada, H., et al. A dipeptidyl peptidase4 inhibitor, sitagliptin, exerts antiinflammatory effects in type 2 diabetic patients. (2013) Metabolism 62(3): 347351.

21. Lee, Y-S., Jun, H-S. Anti-Inflammatory Effects of GLP-1-Based Therapies beyond Glucose Control. (2016) Mediators Inflamm $2016: 3094642$.

22. Boden, G. Obesity and Free Fatty Acids (FFA). (2008) Endoc metab clin North Am 37(3): 635-646.

23. Iwai, T., Ito, S., Tanimitsu, K., et., Glucagon-like peptide-1 inhibits LPS-induced IL-1 beta production in cultured rat astrocytes. (2006) Neurosci Res 55(4): 352-360.

24. Matveyenko, A.V., Dry, S., Cox, H.I., et al. Beneficial endocrine but adverse exocrine effects of sitagliptin in the human islet amyloid polypeptide transgenic rat model of type 2 diabetes: interactions with metformin. (2009) Diabetes 58(7): 1604-1615.

25. Elashoff, M., Matveyenko, A.V., Gier, B., et al. Pancreatitis, pancreatic, and thyroid cancer with glucagon-like peptide-1-based therapies. (2011) Gastroenterology 141(1): 150-156.

26. Nelson, M., Bhandari, N., Wener, J. Sitagliptin-induced pancreatitis - a longer road than expected. (2014) Clin Case Rep 2(4):149-152.

27. Garg, R., Chen, W., Pendergrass, M. Acute Pancreatitis in Type 2 Diabetes Treated With Exenatide or Sitagliptin. (2010) Diabetes Care 33(11): 2349-2354.

28. Faillie, J-L., Azoulay, L., Patenaude, V., et al. Incretin based drugs and risk of acute pancreatitis in patients with type 2 diabetes: cohort study. (2014) BMJ 348: g2780.

29. Information for Healthcare Professionals - Acute pancreatitis and sitagliptin (marketed as Januvia and Janumet). (2009) FDA.

30. Mascolo, A., Rafaniello, C., Sportiello, L., et al. Dipeptidyl Peptidase (DPP) 4 InhibitorInduced Arthritis/Arthralgia: A Review of Clinical Cases. (2016) Drug Saf 39(5): 401407. 
31. MasVidal, A., SantosJuanes, J., EsteveMartinez, A., et al. Psoriasiform eruption triggered by a dipeptidyl peptidase IV inhibitor. Australas J Dermatol. (2012) 53(1): 7072.

32. Amritha, C.A., Kumaravelu, P., Chellathai, D.D. Evaluation of Anti Cancer Effects of DPP-4 Inhibitors in Colon Cancer- An Invitro Study. (2015) J Clin Diag Res 9(12): FC14-FC16.

33. Koehler, J.A, Kain, T., Drucker, D.J. Glucagon-like peptide-1 receptor activation inhibits growth and augments apoptosis in murine CT26 colon cancer cells. (2011) Endocrinology 152(9): 3362-3372.

34. Ohnuma, K., Hatano, R., Morimoto, C. DPP4 in antitumor immunity: going beyond the enzyme. (2015) Nat Immunol 16(8): 791-792.

35. Silva, R.B. da., Laird, M.E., Yatim, N., et al. Dipeptidylpeptidase 4 inhibition enhances lymphocyte trafficking, improving both naturally occurring tumor immunity and immunotherapy. (2015) Nat Immunol 16(8): 850-858.

36. Tseng, C-H. Sitagliptin use and thyroid cancer risk in patients with type 2 diabetes. (2016) Oncotarget.

37. Butler, P.C., Elashoff, M., Elashoff, R.., Gale, E.A. A Critical Analysis of the Clinical Use of Incretin-Based Therapies : Are the GLP-1 therapies safe? (2013) Diabetes Care 36(7): 2118-2125.

38. Aschebrook-Kilfoy, B., Ward, M.H., Sabra, M.M., et al. Thyroid cancer incidence patterns in the United States by histologic type, $1992-2006$. (2011) Thyroid 21(2): 125-134.

39. Bjerre, Knudsen, L., Madsen, L.W., Andersen, S., et al. Glucagon-like Peptide-1 receptor agonists activate rodent thyroid C-cells causing calcitonin release and C-cell proliferation. (2010) Endocrinology 151(4): 1473-86.

40. Hegedus, L., Moses, A.C., Zdravkovic, M., et al. GLP-1 and calcitonin concentration in humans: lack of evidence of calcitonin release from sequential screening in over 5000 subjects with type 2 diabetes or nondiabetic obese subjects treated with the human GLP-1 analog, liraglutide. (2011) J Clin Endocrinol Metab 96(3): 853-860.

41. Gupta, A.K., Verma, A.K., Kailashiya, J., et al. Sitagliptin: anti-platelet effect in diabetes and healthy volunteers. (2012) Platelets 23(8): 565-70. 42. Broxmeyer, H.E., Capitano, M., Campbell, T.B., et al. Modulation of Hematopoietic Chemokine Effects In Vitro and In Vivo by DPP-4/CD26. (2016) Stem Cells Dev 25(8): 575-585.

43. Matheeussen, V., Waumans, Y., Martinet, W., et al. Dipeptidyl peptidases in atherosclerosis: expression and role in macrophage differentiation, activation and apoptosis. (2013) Basic Res Cardiol. 108(3): 350.

44. Fadini, G.P., Avogaro, A. Cardiovascular effects of DPP-4 inhibition: beyond GLP-1. (2011) Vascul Pharmacol 55(1-3): 10-16.

45. Kirby, M., Yu, D., O'Connor, S., et al. Inhibitorselectivity in the clinical application of dipeptidyl peptidase-4inhibition. (2009) Clin Sci(Lond) 118(1): 31-41.

46. Huang, C.Y., Shih, C.M., Tsao, N.W., et al. Dipeptidyl peptidase-4 inhibitor improves neovascularization by increasing circulating endothelial progenitor cells. (2012) Br J Pharmacol 167(7): 1506-1519.

47. Gerger, A., Zhang, W., Yang, D., et al. Common cancer stem cell gene variants predict colon cancer recurrence. (2011) Clin Cancer Res 17(21): 6934-6943.

48. Lowenfels, A.B., Maisonneuve, P., Cavallini, G., et al. Pancreatitis and the risk of pancreatic cancer. International Pancreatitis Study Group. (1993) N Engl J Med 328(20): 1433-1437.

49. Bhanot, U.K., Möller, P. Mechanisms of parenchymal injury and signaling pathways in ectatic ducts of chronic pancreatitis: implications for pancreatic carcinogenesis. (2009) Lab Invest 89(5): 489-497.

50. Vincent, A., Herman, J., Schulick, R., et al. Pancreatic cancer. (2011) Lancet 378(9791): 607-620.

51. Strobel, O., Rosow, D.E., Rakhlin, E.Y, et al. Pancreatic duct glands are distinct ductal compartments that react to chronic injury and mediate Shh-induced metaplasia. (2010) Gastroenterology 138(3): 1166-1177.

52. Yachida, S., Jones, S., Bozic, I., et al. Distant metastasis occurs late during the genetic evolution of pancreatic cancer. (2010) Nature 467(7319): $1114-1117$.

53. Gier, B., Butler, P.C., Lai, C.K., et al. Glucagon like peptide-1 receptor expression in the human thyroid gland. (2012) J Clin Endocrinol Metab 97(1): 121-131.

54. Waser, B., Beetschen, K., Pellegata, N.S., et al. Incretin receptors in non-neoplastic and neoplastic thyroid C cells in rodents and humans: relevance for incretin-based diabetes therapy. (2011) Neuroendocrinology 94(4): 291-301.

55. Liu, Z., Habener, J.F. Glucagon-like peptide-1 activation of TCF7L2-dependent Wnt signaling enhances pancreatic beta cell proliferation. (2008) J Biol Chem 283(13): 8723-8735.

56. Stulc, T., Sedo, A. Inhibition of multifunctional dipeptidyl peptidase-IV: is there a risk of oncological and immunological adverse effects? (2010) Diabetes Res Clin Pract 88(2): 125-131.

57. O'Leary, H., Ou, X., Broxmeyer, H.E. The role of dipeptidyl peptidase 4 in hematopoiesis and transplantation. (2013) Curr opin hematol 20(4): 314-319.

Ommega Online Publisher

Journal of Stem Cell \& Regenerative Biology

Short Title : J Stem Cell Regen Biol
ISSN: 2471-0598

E-mail : stemcells@omegaonline.org

website: www.ommegaonline.org 